\title{
Sterol biosynthesis: 2,3-oxidosqualene analogues
}

\author{
Hisashi Yamamoto* \\ Molecular Catalyst Research Center, Chubu University, Kasugai, Aichi 487-8501, Japan. \\ *Correspondence to: Prof./Dr. Hisashi Yamamoto, Molecular Catalyst Research Center, Chubu University, 1200 Matsumoto-cho, \\ Kasugai, Aichi 487-8501, Japan. E-mail: yamamoto.hisashi@gmail.com
}

How to cite this article: Yamamoto H. Sterol biosynthesis: 2,3-oxidosqualene analogues. Chem Synth 2021;1:7.

https://dx.doi.org/10.20517/cs.2021.12

Received: 19 Aug 2021 Accepted: 20 Aug 2021 Available online: 24 Aug 2021

Academic Editor: Bao-Lian Su Copy Editor: Xi-Jun Chen Production Editor: Xi-Jun Chen

Recently, Krief et al. ${ }^{[1]}$ reported the schizophrenic behavior of oxidosqualene sterol cyclase from pig liver towards 2,3-oxidosqualene analogs bearing two alkyl groups different from a methyl.

The topic of this work is the relatively limited area of squalene cyclization and its rather small aspects of steroid synthesis, but it is the most important biological ${ }^{[2]}$ synthesis of human beings because it is the starting point of steroid synthesis. There are several mysteries of this selectivity, and the topic of this paper is one of the most important steps of biosynthesis. This paper clearly shows the reason for these mysterious issues. The final stage of cyclization generates the necessary rotation of the C-C bond to create the necessary stereochemistry of the product steroid ${ }^{[3]}$. The authors nicely used the different size of the alkyl group to explain the biological synthesis of steroids.

The paper "Schizophrenic behavior of 2,3-Oxidosqualene Sterol Cyclase from pig liver towards 2,3oxidosqualene analogues" is outstanding because it solves the long-standing problem of sterol biosynthesis. Congratulations for a great contribution!

\section{DECLARATIONS}

Authors' contributions

The author contributed solely to the article. 


\section{Availability of data and materials}

Not applicable.

\section{Financial support and sponsorship}

None.

\section{Conflicts of interest}

The author declared that there are no conflicts of interest.

\section{Ethical approval and consent to participate}

Not applicable.

\section{Consent for publication}

Not applicable.

\section{Copyright}

(c) The Author(s) 2021.

\section{REFERENCES}

1. Krief A, Sable R, Ronvaux A, Dumont W, Sandra P, David F. Schizophrenic behavior of 2,3-Oxidosqualene Sterol Cyclase from pig liver towards 2,3-oxidosqualene analogues. Chem Synth 2021;1:6. DOI

2. Thoma R, Schulz-Gasch T, D'Arcy B, et al. Insight into steroid scaffold formation from the structure of human oxidosqualene cyclase. Nature 2004;432:118-22. DOI PubMed

3. Barrett A, Ma T, Mies T. Recent developments in polyene cyclizations and their applications in natural product synthesis. Synthesis 2018;51:67-82. DOI 